\title{
VITAMINES ET STÉRILISATION SOUS VIDE (1)
}

\author{
par \\ JEAN PIEN
}

Ingénieur chimiste (I. C. R.), Docteur ès Sciences. Directeur des Laboratoires des "Fermiers Réunis".

\section{IMPORTANCE DU PROBLEME DE LA CONSERVATION DES MATIÈRES ALIMENTAIRES}

Le problème de la conservation des matières alimentaires présente, de nos jours, une importance considérable.

Grâce aux possibilités offertes par les techniques modernes, qui ont permis de réaliser l'inaltérabilité absolue des aliments les plus variés, nous sommes capables de nous évader en matière de ravitaillement et d'approvisionnement des limites parfois étroites imposées par la nature.

L'usage des conserves a permis, en effet, de résoudre les deux grands problèmes que voici :

\section{$1^{\circ}$ Problème biologique de l'équilibre alimentaire.}

Le régime de l'homme doit comprendre des éléments variés permettant la eonstruction, l'entretien et le fonctionnement de l'organisme par l'apport et l'assimilation de matériaux convenables. Les uns servent à l'édification de la matière vivante, d'autres constituent des sources d'énergie.

Les premières théories de l'alimentation se bornèrent à envisager le rôle énergétique des aliments et la détermination des régimes reposait alors exclusivement sur la eonsidération du nombre de calories qu'ils étaient eapables de fournir à l'organisme. Mais bientôt on s'aperçut que cette théorie était insuffisante. Le rôle des éléments minéraux fut découvert et précisé ; puis enfin celui des vitamines.

A l'heure actuelle, done, on a acquis la certitude que le régime alimentaire doit être équilibré, c'est-à-dire qu'il doit contenir en quantité suffisante et dans un rapport convenable de nombreux éléments variés que l'on peut grouper sous les grandes rubriques suivantes : glucides, protides, lipides, sels minéraux, vitamines.

Mais la répartition de ces substances dans les aliments naturels, végétaux ou animaux, est extrêmement variable. La preuve est faite que l'équilibre alimentaire nécessaire à la vie normale ne peut être réalisé que par un choix judicieux ou, à défaut, par une grande variété de ces aliments. L'usage exclusif de tels ou tels produits

(1) Conférence faite à Nancy le 25 septembre 1936 au Congrès national de I'Industrie laitière. 
naturels, qui pris isolément ne permettent pas cet équilibre nécessaire, conduit à des troubles physiologiques et à des maladies.

Or, il arrive précisément que dans certaines régions et à certaines saisons le nombre de produits naturels est extrêmement réduit. La variété indispensable ne peut être obtenue par les possibilités du lieu ou du moment. A ce titre les conserves, qui permettent de transporter et de stocker les aliments les plus divers, offrent la possibilité de réaliser l'équilibre alimentaire en dépit des conditions parfois sévères imposées par la nature.

\section{$2^{\circ}$ Problème de l'indépendance de l'homme vis-à-vis de la nature.}

L'homme préhistorique ne pouvait se déplacer s'il n'avait acquis la certitude qu'il trouverait au cours ou au terme de son voyage les aliments nécessaires à l'entretien de sa vie. Il ne pouvait songer à emporter avec lui des approvisionnements qui se fussent corrompus.

C'est pourquoi, dès l'antiquité la plus reculée, les hommes ont eu l'idée, puis découvert le moyen de fabriquer des conserves leur permettant par exemple de faire de longs séjours en mer et de s'évader ainsi des nécessités naturelles qui les emprisonnaient.

De nos jours les mêmes besoins sont satisfaits par des moyens. analogues. Mais l'amélioration des techniques, la découverte de procédés de conservation nouveaux, libèrent l'homme plus complètement et lui permettent de vivre dans des conditions et dans des lieux où la nature est incapable de produire les aliments indispensables.

Donc les conserves (au sens le plus large de ce mot) ont joué et jouent de plus en plus dans la vie humaine un rôle de tout premier plan, non seulement en permettant d'équilibrer le régime alimentaire en dépit des conditions locales défavorables, mais encore en fournissant à l'homme le moyen de se libérer de l'emprise de la nature.

On pourrait même dire, sans craindre l'exagération, que les conserves ont joué dans l'Histoire du monde un rôle capital. Sans elles, en effet, sans les viandes séchées ou fumées, sans les salaisons, les enrobages, etc..., comment l'homme eût-il songé à quitter son sol natal ? Comment aurait-il voyagé et surtout exploré dẹs régions inhospitalières, découvert des terres nouvelles ?

Ces considérations sont bien faites pour créer la conviction ou remettre en mémoire l'importance considérable et sans cesse croissante des aliments conservés dans la vie humaine. 


\section{LES PRINCIPES ET LES PROCÉDÉS DE LA CONSERVATION DES DENREES}

On sait qu'il existe de nombreux procédés de conservation des: aliments animaux ou végétaux.

Sans entrer dans le détail de ces procédés, rappelons au moins les grands principes qui sont à la base de la préparation des conserves - principes dont la variété d'application a donné naissance à de nombreuses techniques.

Assurer la conservation d'une matière alimentaire c'est la fixer momentanément ou définitivement dans son état physique, chimique et biologique initial. En d'autres termes e'est s'opposer à sa transformation et en particulier à sa corruption sans toutefois lui faire perdre ses propriétés nutritives.

Il y a plusieurs facteurs capables de provoquer la transformation des matières alimentaires : des agents biologiques comme les microbes et les diastases, des agents chimiques comme l'oxygène, des agents physiques comme la lumière.

Les plus importants et de beaucoup en raison de la variété, de la rapidité et de l'intensité de leur action, sont les microbes.

Conserver une denrée c'est done essentiellement la préserver de l'action des microbes.

En application de ce principe, deux grands groupes de méthodes peuvent être envisagés :

1. Méthodes où l'on se contente de s'opposer à la prolifération des microbes actuellement présents.

2. Méthodes où l'on cherche, au contraire, à détruire les microbes présents tout en s'opposant à de nouvelles pollutions.

Dans le premier groupe, qui est évidemment le moins parfait surtout au point de vue de l'hygiène, on a différents moyens de s'opposer à la prolifération des microbes :

a) En soumettant la denrée à l'action du froid et en l'y maintenant.

Le froid, dans les conditions de la pratique courante, ne détruit pas les microbes mais s'oppose notablement (totalement même suivant les températures et les microbes envisagés) à leur pullulation et à leur action. C'est le principe de la congélation des viandes, de la conservation des fruits à basse température, etc...

b) On peut aussi dessécher complètement la denrée. On sait que les microbes ne peuvent se développer qu'en présence d'un optimum d'humidité.

c) On peut encore créer des conditions dysgénésiques de nature. 
chimique ou physicochimique, en introduisant, dans le milieu que constitue l'aliment, des substances comme le sucre dont la présence à une concentration élevée s'oppose à tout développement bactérien ou autre. D'autres corps comme le sel, ajoutent au mécanisme précédent celui d'un pouvoir antiseptique véritable. Enfin, la création d'une acidité élevée (emploi de l'acide lactique, de l'acide acétique, du vinaigre) joue également un rôle dysgénésique qui préserve la den. rée contre le développement de certaines catégories de microbes.

Toutes ces méthodes ont l'inconvénient de n'assurer qu'une protection assez limitée contre la corruption, attendu que les germes présents ne sont pas détruits, que la denrée n'est pas toujours préservée de l'action d'autres microbes apportés par l'atmosphère et au nombre desquels certains sont capables de s'adapter à vivre en dépit des conditions dysgénésiques que l'on a créées.

Bien plus intéressant est le deuxième groupe qui vise non seulement à détruire radicalement tous les germes présents, mais aussi à protéger l'aliment contre toute pollution ultérieure.

Ces méthodes font appel à l'action de la chaleur.

La plus efficace et la plus sûre est incontestablement la stérilisation, opération qui, par définition, détruit absolument les germes présents dans l'aliment à conserver.

(Notons en passant que la pasteurisation est un cas particulier de cette méthode de conservation par la chaleur. Elle n'a pas l'efficacité de la stérilisation, mais elle ne se propose pas les mêmes buts. La pasteurisation vise seulement à détruire les germes pathogènes. Elle réalise du même coup une protection de quelque durée contre la transformation de l'aliment par certains autres microbes qu'elle a en même temps détruits. Mais elle laisse inévitablement subsister des germes, non pathogènes, mais capables de produire à plus ou moins longue échéance l'altération du produit.)

Donc le procédé de conservation par excellence, celui qui répond de la manière la plus absolue au problème posé est la stérilisation.

$\mathrm{Si}$, au lieu de ne considérer comme facteurs d'altération que les microbes, on les envisage tous (diastases, oxygène, lumière), on s'aperçoit que, là encore, alors que les autres méthodes constituent des palliatifs insuffisants, la stérilisation réalise ou peut réaliser la perfection. En effet, toutes les diastases sont détruites au cours de la stérilisation; et pour peu que la denrée soit enfermée dans un récipient opaque où l'on a fait le vide, o'est-à-dire qu'elle soit protégée contre l'action de la lumière et de l'oxygène, on est amené à constater que ces conditions sont celles qui permettent le mieux d'assurer la conservation de l'aliment. 


\section{$*^{*} *$}

\section{LA VALEUR ALIMENTAIRE DES CONSERVES PAR STÉRILISATION}

Nous n'avons vu jusqu'à présent qu'un aspect du problème : celui de la conservation de la denrée, c'est-à-dire de son maintien dans son état initial.

Mais la définition que nous avons donnée comporte une deuxième partie : l'aliment, avons-nous dit, doit avoir gardé sa valeur et ses qualités nutritives, e'est-à-dire avoir conservé dans leur état initial les einq éléments fondamentaux dont nous avons rappelé en commençant le caractère indispensable : glucides, protides, lipides, sels, vitamines.

Donc, à l'occasion de chaque procédé de conservation, nous: devrions avoir à nous poser les questions suivantes : ces éléments fondamentaux sont-ils conservés ? Sont-ils transformés ? Dans l'affirmative la transformation subie est-elle préjudiciable à la santé humaine ?

Occupons-nous seulement iei du cas de la stérilisation dont nousavons montré qu'elle représentait la perfection en matière de conservation des aliments.

Voyons d'abord rapidement ce qu'il advient des glucides, protides, lipides et des sels dans la stérilisation.

Il est démontré depuis longtemps que dans les conditions où l'on applique dans l'industrie le chauffage des matières alimentaires, ces quatre grands groupes de principes fondamentaux ne sont pas profondément troublés dans leur constitution et dans leur caractère de digestibilité. Sur cet important sujet laissons la parole à M. le Professeur Javillier, de la Faeulté des Sciences de Paris, dont la haute compétence en cette matière fait autorité : "... L'action de la chaleur à ce point de vue, dit-il, est négligeable ou pratiquement nulle. D'ailleurs si, industriellement, on chauffe les matières alimentaires pour en faire des conserves, la cuisinière chauffe, elle aussi, ces mêmes matières à une température un peu moins élevée sans doute, mais pendant un temps plus long que dans l'industrie des conserves. Entre l'aliment conservé par stérilisation et le même aliment transformé par l'art de la cuisine, la différence au point de vue qui nous occupe ici n'est pas grande. »

Nous nous permettrons même d'ajouter que l'action de la chaleur sur certaines protéines en accroît la digestibilité et nombreux sont les hygiénistes qui attribuent à certains aliments, comme le lait, une digestibilité accrue du fait de la stérilisation. (Rappelons d'ailleurs, en passant, que la digestibilité du lait peut être accrue par d'autres procédés, tel celui qui consiste à introduire des hydrates 
de carbone : saccharose, matières amylacées, etc... dans le lait après l'avoir pasteurisé à haute température. L'acidité, dans le cas des babeurres par exemple, joue aussi un rôle très favorable dans la digestibilité du lait.)

Abandonnons donc les protides, les glucides, les lipides et les sels pour en arriver à la capitale question des vitamines.

$$
*^{*} *
$$

LE CAS PARTICULIER DES VITAMINES :

\section{LA STÉRILISATION DÉTRUIT-ELLE OU MODIFIE-T-ELLE} LES VITAMINES ?

Voilà, en somme, posée sans ambiguïté la question capitale, celle qui, en fait, domine pratiquement tout le problème de la conservation des aliments (l'aspect bactériologique étant supposé résolu).

Donnons-en tout de suite la réponse, noūs réservant ensuite d'apporter les justifications nécessaires :

\section{La stérilisation, si elle est effectuée à l'abri de l'oxygène, ne détruit pas les vitamines.}

Cette affirmation, il faut le reconnaître, est assez déconcertante et pour le moins inattendue, car nous étions depuis longtemps tout à fait convaincus de la fragilité des vitamines (de certaines vitamines en tout cas) vis-à-vis de l'action de la chaleur.

Les faits, d'ailleurs, semblaient donner raison à cette eroyance, puisque, par la stérilisation pure et simple, on parvenait à priver réellement les aliments de certaines vitamines et à provoquer sans aucune équivoque les signes des avitaminoses correspondantes chez les animaux de laboratoire.

Mais une série d'études remarquables, peu connues encore et pour la plupart assez récentes, permettent maintenant d'envisager ces questions sous un jour tout nouveau en montrant la distinction capitale qu'il y a lieu de faire entre l'action de l'oxygène et l'action de la chaleur sur les vitamines.

On a aequis la preuve (et nous allons le démontrer pour chacune des vitamines connues) que celles dont on constatait la disparition au cours de la stérilisation ne sont détruites que par l'oxydation; et que, si on les protège contre l'action de l'oxygène pendant le chauffage, elles sont conservées dans une très forte proportion ou même intégralement.

\section{VITAMINE A.}

$$
*^{*} *
$$

C'est la vitamine de croissance. Son absence se traduit par un 
arrêt du développement en volume et en poids de l'animal d'expérience. Une autre conséquence de l'absence de ce facteur est l'augmentation de la sensibilité aux infections (xérophtalmie surtout).

On la rencontre dans la nature sous la forme d'une provitamine, le carotène (pigment jaune très répandu dans le règne végétal associé ou non à la chlorophylle) dans les carottes, les tomates, les bananes, etc... La vitamine A elle-même se forme dans le foie aux dépens du carotène (Travaux de l'Ecole Hollandaise, de Karrer et de ses collaborateurs). Dans le règne animal, en particulier dans les huiles de foie de certains poissons, on rencontre non le carotène mais la vitamine elle-même.

La résistance de cette vitamine à l'action de la température et sa sensibilité à l'oxydation ont été démontrées par Hopkins qui, soumettant du beurre à un chauffage de 4 heures à $120^{\circ}$ en l'absence d'air, constata que sa teneur en facteur $A$ n'était pratiquement pas réduite. Même après 12 heures de stérilisation, la quantité de vitamine A est encore notable. Ce savant a d'ailleurs également montré que, en présence d'air, la destruction de ce facteur est très rapide, même à des températures inférieures.

Woker et WinLimot ont, de même, montré que du beurre chauffé à $88^{\circ}$ pendant 105 minutes en présence d'air, perd son activité vitaminique ; il la perd en 50 minutes si la température est portée à $125^{\circ}$. Si les expériences sont faites à l'abri de l'air, l'activité vitaminique est à peu près totalement respectée.

Cette oxydabilité du facteur A a été confirmée par d'autres auteurs : Peacock, Drummond, Hilditch ; ces savants ont surtout étudié l'oxydation de la vitamine sous l'action de la lumière.

Les huiles se présentent comme ces milieux assez favorables à la eonservation de l'activité vitaminique. Toutefois, le raffinage des huiles d'olive (qui comporte des injections de vapeur surchauffée, des filtrations, c'est-à-dire des conditions favorables à l'oxydation) détruit presque toute activité vitaminique, alors que les huiles brutes sont relativement riches en vitamine A.

Eddy et Kohman qui ont travaillé sur des conserves de légumes ont également montré, qu'en l'absence d'air, la stérilisation ne détruit nullement le facteur $A$. Ainsi, pour ce qui est des épinards, une dose journalière de 26 milligrammes suffit à assurer la croissance normale du rat blanc soumis à un régime dépourvu de vitamines $\mathrm{A}$, qu'il s'agisse d'épinards crus ou d'épinards stérilisés. Ce pouvoir vitaminique des épinards stérilisés était encore intact après 3 ans de conservation.

Même conclusion en ce qui concerne les pois dont la stérilisation n'affecte pas davantage la teneur en facteur A. 
Donc l'agent de destruction de la vitamine $A$ n'est pas la chaleur mais bien l'oxygène. Même à froid, l'oxygène détruit peu à peu cette vitamine. A chaud l'oxydation est accélérée simplement.

Maintenant que l'on connaît bien la formule de la vitamine A que voici (travaux de P. Karrer surtont):

$\mathrm{CH}^{3}$<smiles>[CH]C</smiles>

$\mathrm{C}$<smiles>CC=CC1CCCCC1C=O</smiles>

$\mathrm{CH}^{3}$ $\mathrm{CH}^{3}$ $\mathrm{C}=\mathrm{CH}-\mathrm{CH}=\mathrm{CH}-\stackrel{\text { l }}{\mathrm{CH}}=\mathrm{CH}-\mathrm{CH}^{2} \mathrm{OH}$

on s'explique parfaitement, par des considérations purement chimiques (présence de nombreuses doubles liaisons), cette grande oxydabilité.

\section{VITAMINE B1.}

C'est la vitamine antinévritique. Son absence provoque la polynévrite aviaire ; elle prédispose l'homme à l'atteinte du béri-béri (mais elle n'en serait pas la cause spécifique).

Sa formule est la suivante (travaux de WiLliams, 1935) :

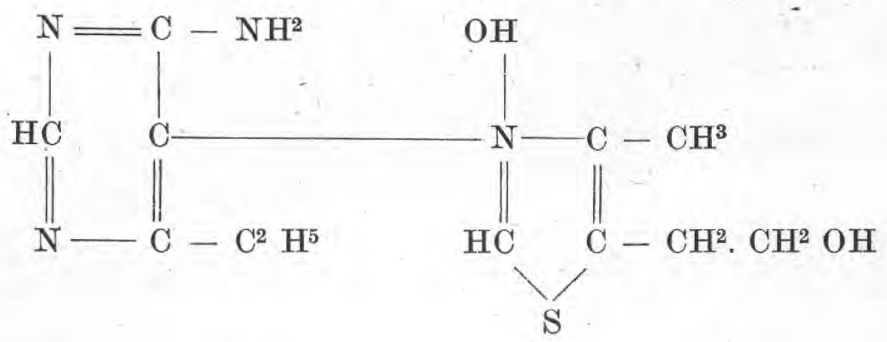

Très répandue dans le règne végétal, cette vitamine se rencontre dans les germes des céréales, les légumes à feuilles vertes, la tomate et surtout dans la levure qui en est la source la plus riche. Les aliments animaux (lait, viande, poissons) en contiennent très peu.

La résistance à la chaleur de la vitamine B1 a fait l'objet de nombreuses discussions en raison de l'extrême difficulté de l'étudede ce facteur. La complexité de la vitamine B, maintenant dissociée en facteur B1 et facteur B2, a été la cause d'erreurs et de contra. dictions de la part de plusieurs auteurs étrangers (1).

(1) Certains auteurs ont même eru pouvoir distinguer jusqu'à six vitamines dans la vitamine B d'autrefois. 
A l'heure actuelle la question parait définitivement réglée.

Les travaux de Daniels et de Mac CluRg, puis ceux de Eddy et КонмаN ont montré que la stérilisation n'entrâ̂ne pas une diminution notable de la teneur en vitamine $\mathrm{B} 1$ des haricots ou des pois.

Scotti-Fogriéni a montré d'autre part que le facteur B du son du riz est détruit en 30 minutes à $120^{\circ}$ quand le chauffage est effectué en présence d'air, alors que la destruction est nulle à $134^{\circ}$ pendant 1 heure et demie quand le son est placé dans un récipient hermétiquement clos.

Portier, Mme Randoin, Simonnet ont montré que le facteur B est particulièrement résistant à l'action de la chaleur. "Sa destruction, écrivent ces savants, n'est complète que si l'on maintient la substance traitée pendant plusieurs heures à $130-140^{\circ}$. ")

\section{VITAMINE B2.}

C'est la vitamine anti-pellagreuse ou d'intégrité dermique. Son absence détermine la pellagre. Elle joue également un rôle très important dans l'utilisation des glucides.

Sa formule est la suivante (travaux de KUHN) :

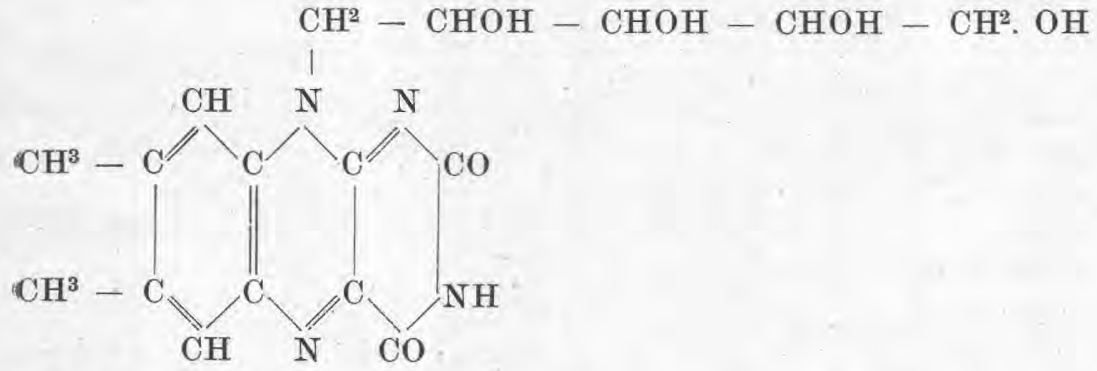

Extraite à l'état pur c'est un beau corps jaune à fluorescence verte qui correspond assez bien à la lactoflavine du lait qui en contient environ 1 gramme pour 10.000 litres (KUнN, 1934).

Cette vitamine se rencontre dans le lait, la levure, les légumes à feuilles vertes, la viande, la tomate. Certains aliments la renferment sans posséder le facteur BI (blane d'œuf par exemple). Pour d'autres (germe de blé) e'est l'inverse.

La vitamine B2 est très résistante à la chaleur, plus résistante que le facteur B1. (Cette propriété a été mise à profit pour la séparation de ces deux vitamines.) Elle n'est pas détruite par un chauffage de 24 heures à $120^{\circ}$.

La meilleure preuve qu'on puisse donner de cette résistance est le résultat curatif indiscutable obtenu par GoLdBerger dans le traitement de la pellagre par l'utilisation de certaines conserves stérilisées comme source de vitamine $B 2$. 


\section{VITAMINE C.}

C'est la vitamine antiscorbutique. Son absence détermine le scorbut (spontané ou expérimental).

Cette vitamine se rencontre surtout dans le jus de citron et d'orange, dans la tomate, le chou. Presque tous les légumes et les fruits en contiennent. Les aliments d'origine animale (lait, viande) en contiennent peu.

La constitution chimique de cette vitamine est la suivante (travaux de Micheel, d'Euler, de Cox, de Hirst, de ReichsTEIN, etc...) :

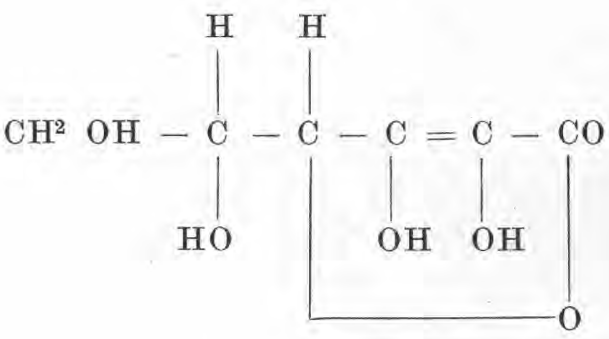

La sensibilité de ce facteur vis-à-vis de l'oxydation est très grande. C'est l'ignorance de cette propriété qui faisait dire aux premiers expérimentateurs que la vitamine $\mathrm{C}$ était très peu résistante à l'action de la chaleur. Le chauffage en présence d'air, en effet, la détruit en quelques minutes même à des températures assez basses. En revanche, en l'absence stricte d'oxygène, elle est stable à la chaleur (1).

Un savant hongrois, Szent-Gyorgi, a isolé il y a peu d'années la vitamine C à l'état pur (acide hexuronique) et lui a donné, depuis, le nom d'acide ascorbique. Ce corps est éminemment oxydable et facilement détruit par la chaleur en présence d'oxygène. Mais en l'absence d'oxygène ou d'air il résiste parfaitement à l'action de la chaleur.

Ce qui est vrai de l'acide ascorbique l'est à peu près identique-

(1) Un cas particulier très curieux doit être signalé comme conséquence de ce prin. cipe : la dessiccation du lait par pulvérisation, même en présence d'air, conserve la vitamine $\mathrm{C}$ (et à fortioriles autres puisque celle-ci est la plus fragile) ainsi qu'il résulte des travaux de RENNER, de STOERR, etc.

Peut-être y a-t-il lieu d'admettre que les particules de lait (même dans le cas du lait écrémé) sont suffisamment protégées contre l'action de l'oxygène par la matière grasse (même à l'état de traces).

D'ailleurs, la pulvérisation du lait étant instantanée dans ces techniques, le "chauf. fage » en présence d'air se trouve réduit à une durée extrêmement courte qui ne per mettrait pas l'oxydation des vitamines. 
ment des aliments qui le renferment. Il y a lieu toutefois de faire une légère distinction à cet égard entre les légumes et les fruits.

Dans le eas des légumes (en raison sans doute de l'oxygène conservé dans les cellules végétales) on constate une certaine perte de l'activité vitaminique par la stérilisation. Mais si le chauffage a lieu à l'air (même à une température plus basse, $100^{\circ}$ par exemple) la perte de vitamine $C$ est 5 fois plus grande, ainsi qu'il résulte des travaux de EDDx et KoHMAN. Comme les légumes sont rarement consommés à l'état cru, mais cuits à $100^{\circ}$ en présence de l'air, on constate done que la stérilisation présente une supériorité incontestable sur la cuisson habituelle.

Dans le cas des fruits, qui sont plus souvent consommés à l'état cru, la question présente une importance capitale. Or, il résulte des travaux de KонмаN et de ses collaborateurs que si on prend la précaution de se débarrasser de l'oxygène intracellulaire (par immersion préalable dans l'eau salée pendant 16 heures, de manière à permettre la consommation de eet oxygène résiduel par respiration intracellulaire) la stérilisation ne diminue pas la quantité de facteur C présent, alors que cette opération effectuée sans immersion préalable (et à fortiori un chauffage à l'air libre) font disparaître pratiquement toute activité antiscorbutique.

D'ailleurs, même à froid, des fruits conservés à l'air (pommes crues gardées plusieurs mois en frigorifique) perdent peu à peu leur facteur C.

Des expériences concernant la stérilisation ont été effectuées sur. des pommes, des fraises, des tomates. Elles montrent toutes que si on parvient à se protéger complètement de l'action de l'oxygène pendant la stérilisation (et aussi au cours de certaines opérations préalables telles que le dépulpage des tomates) on conserve intégralement le facteur C. Expérimentalement cela signifie que pour prévenir le scorbut chez le cobaye soumis à un régime scorbutigène on est amené à introduire dans la ration une quantité identique de fruits crus ou de fruits conservés, preuve indiscutable que la stérilisation n'a pas diminué, dans ces conditions, le pouvoir antiscorbutique des aliments étudiés.

\section{VITAMINE D.}

C'est la vitamine antirachitique; elle joue un rôle important dans le métabolisme du phosphore et du calcium et dans la formation du tissu osseux. Son absence détermine la maladie du rachitisme,

Cette vitamine se rencontre dans la nature principalement dans les huiles de foie de poissons et, en quantité moindre, dans le lait, le beurre, les œufs. Il faut considérer également comme une 
source indirecte mais importante de vitamine $\mathrm{D}$ la lumière solaire qui, par ses radiations ultra-violettes, transforme l'ergostérol contenu dans certaines graisses en vitamine antirachitique.

Voici la formule chimique la plus probable de la vitamine D (récents travaux de LETTRÉ) :

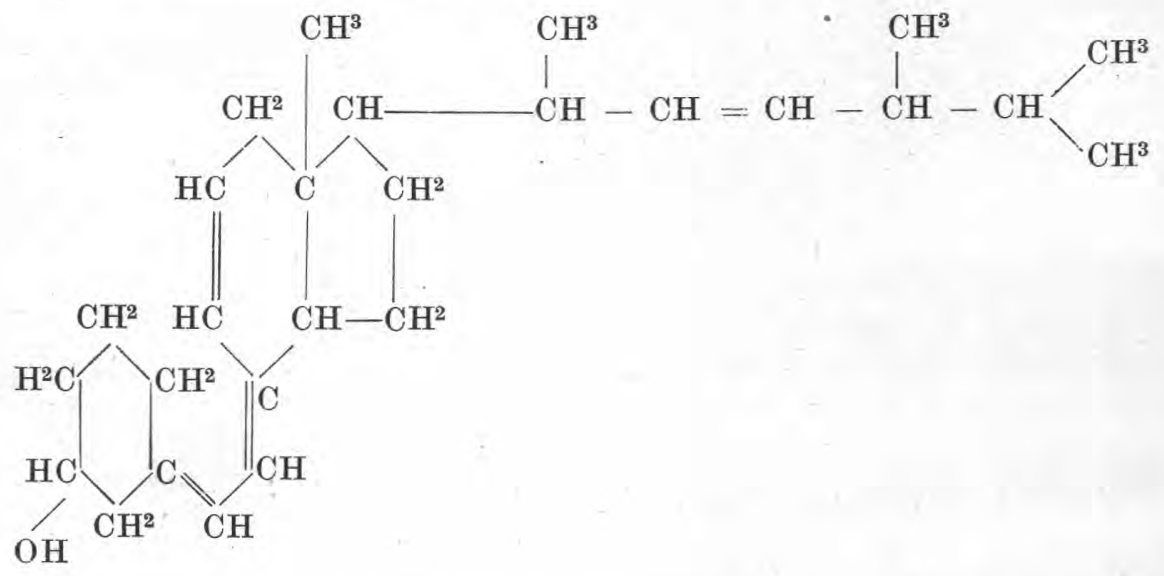

La résistance à la chaleur de cette vitamine a été démontrée par les travaux de Mc. Collum, de Goldblatt, de Zilva. Ces auteurs ont montré que l'huile de foie de morue peut être chauffée pendant 12 heures à $100^{\circ}$ à l'air sans constater aucune diminution de son pouvoir antirachitique.

Mme Randorn a d'ailleurs montré que le facteur D n'est pratiquement pas détruit par un chauffage de 4 heures à l'autoclave.

Personnellement, nous avons acquis la preuve, au cours de travaux qui ne sont pas encore publiés, que le pouvoir antirachitique du lait n'est pas diminué par la stérilisation.

\section{VITAMINE E.}

C'est la vitamine de reproduction. Son absence provoque la stérilité. On la rencontre dans les huiles de germes de céréales. Certains végétaux comme la laitue (dans les parties vertes) en contiennent aussi notablement.

La haute résistance à la chaleur de cette vitamine est également prouvée.

Il résulte des travaux de Evans et Bishop, de Sure, que les légumes qui contiennent cette vitamine la conservent intégralement même après 1 heure et demie de séjour à l'autoclave à $120^{\circ}$. 
En résumé on se rend compte qu'il y a lieu de classer les vitamines en deux groupes:

Les vitamines $\mathrm{B} 2, \mathrm{D}, \mathrm{E}$, qui sont très résistantes à la chaleur, même en présence d'oxygène.

Les vitamines $\mathrm{A}, \mathrm{B} 1, \mathrm{C}$, qui ont semblé tout d'abord ne pas résister à l'action de la chaleur, mais dont il a été prouvé que la cause de la destruction était leur sensibilité parfois très grande à l'oxydation. Si on prend soin de protéger ces vitamines contre l'action de l'oxygène, on s'aperçoit qu'elles résistent, elles aussi, à l'action de la chaleur et qu'elles ne sont pas détruites dans les conditions thermiques de la stéritisation industrielle.

$$
*^{*} *
$$

\section{EXPÉRIENCES SYNTHÉTIQUES D'ALIMENTATION PROLONGÉE A L'AIDE DE RÉGIMES STÉRILISÉS}

Nous avons, dès maintenant, tous les :éléments nous permettant de tirer la conclusion de cet exposé. Toutefois nous croyons intéressant de signaler auparavant deux séries de travaux de la plus haute importance qui sont comme une synthèse et un couronnement de tout ce qui précède.

Les études rappelées brièvement ci-dessus, en effet, n'ont montré que le résultat d'expériences effectuées isolément sur telle ou telle vitamine en prenant comme test la prévention ou la guérison de lésions caractéristiques.

Il est utile, alors, de se demander ce qu'il advient de la santé générale, du développement, de la résistance aux infections, de la vie en un mot d'animaux nourris exclusivement avec des aliments stérilisés et cela pendant plusieurs mois ou plusieurs années, c'est-àdire en expérimentant sur plusieurs générations consécutives.

Ce programme a été réalisé par EDDy et КонмAN à l'étranger et plus récemment en France par le $\mathrm{D}^{\mathrm{r}}$ Machebeuf, H. Cheftel et $\mathrm{M}^{\mathrm{me}}$ ThüLLот.

Ces expériences ont été couronnées d'un succès complet.

EDdy et KoHman ont obtenu une croissance normale du rat et du cobaye. Quand ils ont fait connaître l'état de leurs travaux, ils en étaient à la huitième génération de cobayes et à la troisième génération de rats avec des animaux en tous points normaux.

Le $\mathrm{D}^{\mathrm{r}} \mathrm{M}_{\mathrm{ACH}} \mathrm{B}$ EUF et ses collaborateurs ont obtenu des résultats aussi satisfaisants :

De mai 1931 à septembre 1933 (date de publication de leur mémoire) ils ont obtenu 6 générations de rats avec 157 naissances. Les courbes de croissances ont été parfaitement normales; les radio- 
graphies montrent une formation parfaite du squelette même après 5 générations.

D'avril 1932 à juillet 1933 les cobayes ont fourni 3 générations avec 42 naissances; courbes de croíssance normales ; état satisfaisant des animaux.

Tous ces animaux ont reçu eomme alimentation exclusive des conserves en boîtes de : légumes, viande, poissons, fruits... les plus divers.

Le Dr MacheB de leurs recherches en une brochure intitulée "La valeur alimentaire des conserves" (septembre 1933) (Gauthier-Villars, éditeur).

C'est dans cette étude (dont nous ne saurions trop conseiller la lecture) que nous avons puisé la majeure partie de notre documentation.

Qu'il nous soit permis de signaler également l'état actuel de nos propres travaux dans ce même domaine.

Nous avons soumis un élevage de rats blanes depuis près de 12 mois à une alimentation composée de légumes variés, de viande, de poissons, de fromages, de lait.

La moitié de l'élevage reçoit tous ces aliments à l'état frais. L'autre moitié reçoit les mêmes aliments stérilisés de la manière suivante : les aliments végétaux sont d'abord immergés 24 heures dans l'eau pour faire disparaître l'oxygène intracellulaire comme dans le cas des expériences de KoHman. Puis les récipients contenant tous les aliments sont soumis à l'action prolongée d'un courant de gaz carbonique, ce qui élimine pratiquement tout l'oxygène atmosphérique. Les récipients sont hermétiquement clos et passés à l'autoclave à $120^{\circ}$ pendant 20 minutes.

La litière des animaux est également stérilisée, de manière à enlever tout doute sur l'éventualité d'un apport, si faible soit-il, d'un aliment ou d'une substance quelconque n'ayant pas subi l'action de la chaleur.

Ces expériences doivent être poursuivies pendant plusieurs années. Néanmoins nous pouvons dès à présent donner les résultats qui concernent la première génération :

\section{Résumé de la courbe de croissance.}

On part en janvier 1936 avec 10 rats dans chaque série d'un poids unitaire moyen de 70 grammes pour ceux qui recevront le régime frais et de 68 grammes 5 pour ceux qui recevront le même régime stérilisé.

On arrête la première génération 5 mois plus tard et on aboutit à un poids unitaire moyen de 288 grammes dans la série régime frais et 277 grammes dans la série régime stérilisé. 
Il faut done noter que le régime frais a fourni un peu plus de poids vif que le régime stérilisé. Faut-il en conclure que le régime stérilisé est moins favorable au développement que le régime frais ? Cette conclusion serait peut-être prématurée car les animaux soumis au régime stérile consomment une quantité totale d'aliments un peu inférieure à celle qui est consommée dans l'autre série, en raison d'une différence de saveur, d'aspect, de consistance à laquelle les rats ne sont nullement insensibles. Les aliments stérilisés étant consommés en quantité un peu moindre, surtout au début, il est assez normal qu'un léger retard soit constaté, dans le poids.

\section{COURBES DU POIOS MOYEN DUUN RAT PENDANT 5 MOIS D'EXPÉRIENCE.}

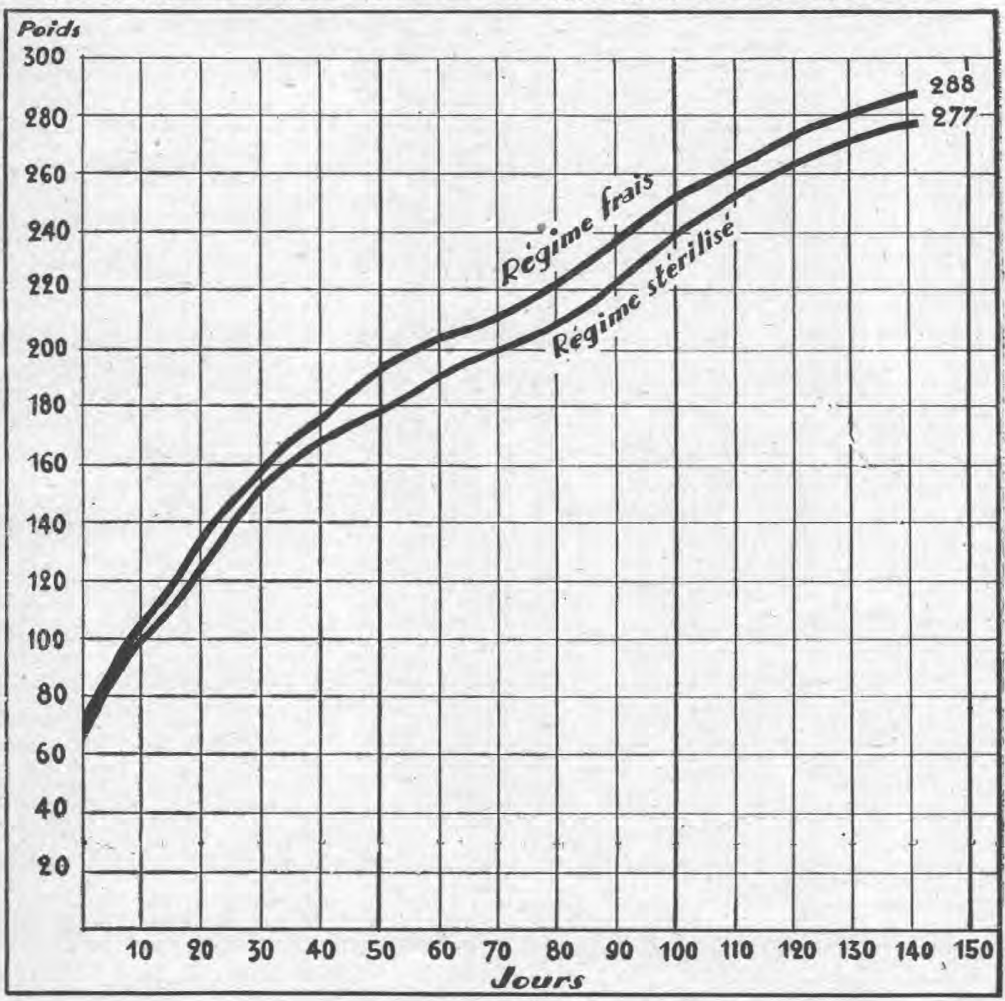

Ce qui importe, à notre avis, dans ces courbes, c'est leur allure. Si elles étaient constamment divergentes le pronostic serait grave. Or, on constate, au contraire, qu'après avoir légèrement divergé, sans doute pour la raison qui vient d'être indiquée, ces courbes ont tendance à se rapprocher, puis restent parallèles pendant les derniers mois de l'expérience. A ce moment on a acquis la preuve 
que l'accélération de l'aceroissement (la dérivée de la fonction) est la même dans les deux cas, ce qui corréspond à des états physiologiques identiques au moins en ce qui concerne les poids.

\section{$2 \circ$ Aptitude à la reproduction.}

Dans le cours de l'expérience, une deuxième, puis, plus récemment une troisième génération ont été créées en accouplant entre eux certains mâles et certaines femelles à l'intérieur de chaque série alimentaire. Bien entendu ces femelles ont été éliminées dans la détermination des courbes de poids.

Le nombre de jeunes nés après une durée de gestation normale a été le suivant (deuxième et troisième générations) :

Série soumise au régime frais : 41 jeunes pour 6 femelles.

Série soumise au régime stérile : 57 jeunes pour 6 femelles.

Ce résultat montre que la fécondité n'a pas été altérée par le régime stérilisé puisque c'est dans cette série qu'on a le plus de naissances.

Bien entendu cette notion" est à contrôler sur de nombreuses générations successives.

\section{$3^{\circ}$ Etat de santé. Résistance aux infections.}

Aucune maladie n'a été enregistrée. Aucun signe d'avitaminose quelconque n'a pu être noté jusqu'à présent (troisième génération). L'aspect des animaux, leur port, leur pelage, leurs habitudes, la vivacité des jeunes, etc., ont été absolument identiques dans les deux séries.

Donc, jusqu'à présent (et bien qu'il nous soit interdit de conclure puisque l'expérience ne dure que depuis dix mois), nous pouvons dire que nos premières observations corroborent pleinement celles de Eddy et Kohman, et celles du Dr Machebeur et de ses collaborateurs.

Nous ne saurions donc mieux faire pour tirer l'enseignement des études synthétiques prolongées de ces auteurs que de citer la conclusion du travail du $\mathrm{D}^{\mathrm{r}}$ MACHEBEUF :

"En conclusion, nous voyons que des animaux aussi sensibles " aux avitaminoses que le sont le rat et le cobaye peuvent sub"sister, se développer et se reproduire parfaitement lorsqu'ils "sont alimentés uniquement par des conserves en boîtes de fer"blanc, à l'exclusion de tout aliment frais ou cru. "

La conclusion d'une étude de M. le Professeur JAvilLien sup le même sujet (étude à laquelle nous avons également fait de nombreux emprunts surtout en ce qui concerne la constitution chimique des vitamines) est non moins nette : 
"Nous conclurons done que la valeur du régime alimentaire des conserves est égale ou voisine de celle des aliments frais cuisinés et, même, j'ose dire que cette valeur alimentaire est parfois supérieure à celle d'aliments cuisinés. "

$$
*^{*} *
$$

\section{CAS DE L'ALIMENTATION HUMAINE}

Mais, dira-t-on, ces conclusions si précises et si importantes soient-elles ne sont basées que sur des résultats d'expériences effectuées sur les animaux.

Ces conclusions sont-elles valables pour l'homme?

Or, précisément, l'expérience a été également faite.

En 1932-1933 la mission polaire CHARCOT (dont nous avons appris récemment la si tragique disparition) a emporté une série de conserves préparées sous la direction de MM. MACHEBGeUf et CHefteL (conserves de viandes, de légumes, de poissons, de fruits, de lait, de fromages, de beurre, etc...).

Voici un extrait du compte rendu de cette expérience sensationnelle :

"Les résultats d'alimentation furent des plus satisfaisants : aucun accident scorbutique ne fut observé et cela malgré une recherche très soigneuse des moindres symptômes. Pourtant on ne fit jamais usage ni de citrons frais, ni de purée de tomate, ces produits ayant été gardés comme réserve.

"Personne ne ressentit le besoin d'aliments frais après un temps si long de consommation de conserves.

"D'autre part, aucun accident d'intoxication par les conserves n'a été constaté.

"Cette expérience semble bien prouver qu'un régime basé uniquement sur des conserves et dépourvu d'aliments frais ne présente aucun inconvénient pour l'alimentation de l'homme, même pendant une période assez longue, à condition qu'il soit très convenablement équilibré et soigneusement varié. "

$$
\text { ** * }
$$

\section{CONCLUSIONS}

La conclusion qui se dégage de cet exposé semble pouvoir être la suivante :

Les vitamines actuellement connues sont pratiquement résistantes à l'action de la chaleur dans les conditions de la stérilisation, si l'on prend soin de les protéger de l'action de l'oxygène. 
Autrement dit : la stérilisation dans le vide ou dans une atmosphère non oxydante (azote, gaz carbonique) permet la conservation du pouvoir vitaminique des aliments.

Il est inutile de souligner l'intérêt fondamental de cette donnée. Son incidence médicale, économique, industrielle est de la plus haute importance par les conséquences pratiques qu'elle est susceptible de comporter dans l'avenir.

$$
*^{*} *
$$

\section{APPLICATION DES DONNÉES PRÉCÉDENTES AU CAS DU LAIT}

Messieurs, si ma conférence devait se terminer sur ces mots, on pourrait sans doute me reprocher d'avoir introduit cette question dans un congrès destiné à l'étude des questions laitières.

Mais je pense, au contraire, que ce sujet et cette conclusion doivent nous intéresser au plus haut point.

L'étude de la stérilisation du lait - et de son influence sur la teneur en vitamines de cet aliment - n'est, en effet, qu'un cas particulier important de tout ce qui précède.

Les griefs formulés contre le lait stérilisé, parfaitement fondés dans le cas de stérilisations en présence d'air qui ne respectent pas certaines vitamines, tombent, en raison de tout ce qui vient d'être dit, si la stérilisation est faite dans le vide ou dans un gaz inerte.

Il est hors de doute - sous réserve bien entendu de nouvelles études et de nouvelles confirmations dans l'avenir - que l'extension de ces notions au cas du lait serait capable d'avoir de profondes répercussions dans notre industrie.

Et il ne s'agit pas seulement ici du cas de l'alimentation des enfants. Cette question vise aussi l'alimentation humaine en général.

\section{Cas de l'alimentation des enfants.}

Dans le cas des nourrissons et des enfants, la question du lait doit s'étudier au triple point de vue de la diététique, de l'hygiène bactériologique et des vitamines.

a) La diététique (dans le domaine qui nous occupe ici) s'intéresse surtout à l'influence du chauffage sur les éléments du lait: nulle sur les glucides et les lipides, cette incidence paraît favorable dans le cas des protides ainsi que nous l'avons dit. De nombreux pédiâtres reconnaissent à l'heure actuelle aux laits chauffés (pasteurisés) et en particulier aux laits stérilisés, une plus grande digestibilité de la caséine et peut-être une meilleure utilisation. Il faut sans doute mettre de côté certaines inappétences et même certaines répulsions vis-à-vis du lait stérilisé qui tiennent sans doute plus au fait d'un changement brutal de régime qu'à un accident biologique. Les mê- 
mes faits ont d'ailleurs été observés en sens inverse ; et il apparaît que rien de semblable ne serait constaté si, avant de passer brusquement à une ration complète en lait stérilisé, on faisait précéder ce nouveau régime d'une alimentation mixte à dosage progressif.

Quoi qu'il en soit, des pédiâtres renommés n'hésitent pas à dire que l'alimentation des enfants à base de lait stérilisé, non seulement n'a pas d'inconvénients diététiques, mais est quelquefois désirable.

b) L'hygiène bactériologique, elle, est incontestablement en faveur du lait stérilisé qui présente les plus absolues garanties non seulement pour le lait lui-même (ce que d'autres techniques assurent déjà) mais aussi et surtout pour les conditions de la distribution à l'enfant.

Le fait pour une maman inexpérimentée d'introduire du lait déjà parfaitement sain dans une casserole ou dans un biberon qui le sont moins..., est souvent l'occasion pour le nourrisson de souffrir de troubles intestinaux. La livraison de biberons stérilisés à l'avance, de conservation indéfinie, contenant le lait au taux de dilution et de sucre correspondant aux 10 ou 15 cas les plus typiques d'âge et d'état de santé, résoudrait sans doute le problème de l'alimentation infantile.

c) Enfin, le troisième point de vue, celui des vitamines - source de l'objection la plus grave contre le lait stérilisé - , il a été examiné tout au long de cette étúde.

Il semble donc que l'alimentation des nourrissons, dans l'avenir, puisse s'orienter sur la voie du lait stérilisé ; ou, du moins, il semble bien que l'étude de cette question doit être entreprise maintenant sous un jour tout à fait nouveau.

\section{$2^{\circ}$ Cas de l'alimentation humaine en général.}

Reste la question capitale, celle qui vise l'alimentation humaine elle-même à l'aide de lait stérilisé.

A l'heure actuelle, cette question est encore du domaine de l'utopie..., mais peut-être comme l'était celle de la pasteurisation il y a cinquante ans.

Ce problème est-il industriellement réalisable? Oui. On sait faire de la stérilisation en continu à grand débit et à l'abri de l'air. On peut boucher aseptiquement et sous vide, industriellement. Bien entendu, cela suppose la vente du lait en flacons de verre. Mais nous y venons pour le lait pasteurisé. Enfin le lait stérilisé en continu, ou autrement, peut être parfaitement blanc et sans aucune saveur désagréable.

Quels avantages présenterait la vente du lait stérilisé ? Ils sautent aux yeux : le problème de la conservation du lait serait rayé de la 
liste de nos soucis et de nos difficultés. L'approvisionnement des villes en lait ne serait plus soumis à d'impérieuses conditions d'heures et de délais. Le problème du froid en laiterie n'existerait pour ainsi dire plus. Le transport, les livraisons se feraient dans des conditions plus économiques puisque la distribution n'aurait plus à être quotidienne. La possibilité du stockage, soit dans nos usines, soit au stade de la vente, soit chez le consommateur, modifierait entièrement l'aspect de nos industries. Notons encore que les excédents d'été pourraient être stérilisés, conservés pour la vente en hiver et qu'il pourrait en résulter d'heureuses conséquences économiques.

Donc, non seulement le problème comporte une solution, mais cette solution présenterait de très sérieux avantages pour l'industrie laitière.

Certes, ce sont là des vues d'avenir de réalisation non immédiate.

Elles peuvent soulever de nombreuses objections.

Mais ne manquons pas de méditer ceci : autrefois, le Corps Médical, l'Académie de Médecine, le Conseil Supérieur d'Hygiène, seseraient opposés, à cause précisément de la destruction des vitamines, à la généralisation de l'usage du lait stérilisé dans l'alimentation humaine, comme dans l'alimentation infantile. Aujourd'hui cette objection tombe. Elle tombe non pas devant des idées ou des: arguments, mais devant des faits.

Comme d'autre part ces mêmes Hautes Assemblées, et avec elles le Corps Médical tout entier, verront surtout dans la stérilisation $l a$ solution intégrale du problème de l'hygiène du lait, il est à penser que le pas qui sépare la pasteurisation obligatoire de la stérilisation obligatoire sera beaucoup plus rapidement franchi que celui qui séparait la vente entièrement libre du lait cru de la vente, devenue partiellement obligatoire en fait, du lait pasteurisé.

$$
*^{*} *
$$

Que de nouvelles recherches soient désirables, que l'on exige des vérifications, des contrôles, on le conçoit. Que ces idées soulèvent des difficultés de réalisation pratique; que, à certains autres points. de vue, elles puissent être discutées, on le comprend. Mais nous restons convaincus que, d'une part, l'autorité des savants dont nous avons invoqué le témoignage, d'autre part, le souci toujours croissant des Pouvoirs publics d'améliorer l'hygiène du lait, nous font. le devoir de ne pas rejeter cette éventualité comme une pure utopie.

C'est, au contraire, une idée d'avenir que nous avons cru bon d'exposer pour la première fois dans un congrès de laiterie. 